\title{
Lens partition function, pentagon identity, and star-triangle relation
}

\author{
Deniz N. Bozkurt $\odot,{ }^{1, *}$ Ilmar Gahramanov $\odot,{ }^{2,3,4, \dagger}$ and Mustafa Mullahasanoglu ${ }^{2, \$}$ \\ ${ }^{1}$ Department of Physics, Koc University, 34450 Sariyer, Istanbul, Turkey \\ ${ }^{2}$ Department of Physics, Bogazici University, 34342 Bebek, Istanbul, Turkey \\ ${ }^{3}$ Institute of Radiation Problems, Azerbaijan National Academy of Sciences, \\ B.Vahabzade St. 9, AZ1143 Baku, Azerbaijan \\ ${ }^{4}$ Department of Mathematics, Khazar University, Mehseti St. 41, AZ1096 Baku, Azerbaijan
}

(Received 23 November 2020; accepted 13 April 2021; published 23 June 2021)

\begin{abstract}
We study the three-dimensional lens partition function for $\mathcal{N}=2$ supersymmetric gauge dual theories on $S^{3} / \mathbb{Z}_{r}$ by using the gauge/Yang-Baxter equation correspondence. This correspondence relates supersymmetric gauge theories to exactly solvable models of statistical mechanics. The equality of partition functions for the three-dimensional supersymmetric dual theories can be written as an integral identity for hyperbolic hypergeometric functions. We obtain such an integral identity which can be written as the star-triangle relation for Ising type integrable models and as the integral pentagon identity. The latter represents the basic 2-3 Pachner move for triangulated 3-manifolds. A special case of our integral identity can be used for proving orthogonality and completeness relation of the Clebsch-Gordan coefficients for the self-dual continuous series of $U_{q}(\operatorname{osp}(1 \mid 2))$.
\end{abstract}

DOI: 10.1103/PhysRevD.103.126013

\section{INTRODUCTION}

The recent progress in gauge/Yang-Baxter equation (YBE) correspondence has lead to remarkable connections between supersymmetric gauge theories, integrable models of statistical mechanics, and special functions. The main idea of the correspondence is that the supersymmetric duality for gauge theories leads to the integrability for spin lattice models, see [1,2] for a review and references therein. This interplay between supersymmetric theories and integrable models enables us to generate new solutions to the star-triangle relation which is a special form of the YangBaxter equation, ${ }^{1}$ see e.g., [3-9]. The star-triangle relation is a sufficient condition for integrability of Ising-type lattice models $[10,11]$. It seems that the gauge/YBE correspondence gives a general method to construct solutions to the star-triangle relation.

In this work we use the gauge/YBE correspondence to obtain the star-triangle relation and the pentagon identity in

\footnotetext{
*dbozkurt16@ku.edu.tr

†ilmar.gahramanov@boun.edu.tr

${ }^{\ddagger}$ mustafa.mullahasanoglu@ boun.edu.tr

${ }^{1}$ There are Interaction-Round-a-Face Models (IRF) and vertex models studied in the context of gauge/YBE correspondence; in the paper we will only discuss Ising-type models.
}

Published by the American Physical Society under the terms of the Creative Commons Attribution 4.0 International license. Further distribution of this work must maintain attribution to the author(s) and the published article's title, journal citation, and DOI. Funded by SCOAP ${ }^{3}$. terms of hyperbolic hypergeometric functions. From the gauge theory side we consider the partition functions of $\mathcal{N}=2$ supersymmetric dual gauge theories on $S^{3} / \mathbb{Z}_{r}$. Such lens partition functions were studied from different aspects in several papers, see, e.g., [9,12-21].

As the main result of the paper one may regard the following hyperbolic hypergeometric identity:

$$
\begin{aligned}
& \frac{1}{r \sqrt{-\omega_{1} \omega_{2}}} \sum_{y=0}^{[r / 2]} \epsilon(y) e^{\frac{\pi i C}{2}} \int_{-\infty}^{\infty} d z \prod_{i=1}^{3} \gamma^{(2)}\left(-i\left(a_{i}-z\right)\right. \\
& \left.-i \omega_{1}\left(u_{i}-y\right) ;-i \omega_{1} r,-i \omega_{1}-i \omega_{2}\right) \\
& \times \gamma^{(2)}\left(-i\left(a_{i}-z\right)-i \omega_{2}\left(r-u_{i}+y\right) ;-i \omega_{2} r,-i \omega_{1}-i \omega_{2}\right) \\
& \times \gamma^{(2)}\left(-i\left(b_{i}+z\right)-i \omega_{1}\left(v_{i}+y\right) ;-i \omega_{1} r,-i \omega_{1}-i \omega_{2}\right) \\
& \times \gamma^{(2)}\left(-i\left(b_{i}+z\right)-i \omega_{2}\left(r-\left(v_{i}+y\right)\right) ;-i \omega_{2} r,-i \omega_{1}-i \omega_{2}\right) \\
& =\prod_{i, j=1}^{3} \gamma^{(2)}\left(-i\left(a_{i}+b_{j}\right)-i \omega_{1}\left(u_{i}+v_{j}\right) ;-i \omega_{1} r,-i \omega_{1}-i \omega_{2}\right) \\
& \left.\gamma^{(2)}\left(-i\left(a_{i}+b_{j}\right)-i \omega_{2}\left(r-u_{i}-v_{j}\right) ;-i \omega_{2} r,-i \omega_{1}-i \omega_{2}\right)\right),
\end{aligned}
$$

with the balancing conditions $\sum_{i} a_{i}+b_{i}=\omega_{1}+\omega_{2}$ and $\sum_{i} u_{i}+v_{i}=0$. For the exponential term, we have $C=-2 y+\left(u_{1}+u_{2}+u_{3}-v_{1}-v_{2}-v_{3}\right)$, the $\epsilon(y)$ function is defined as $\epsilon(0)=\epsilon\left(\left\lfloor\frac{r}{2}\right\rfloor\right)=1$ and $\epsilon(y)=2$ otherwise. The hyperbolic gamma functions is defined as 


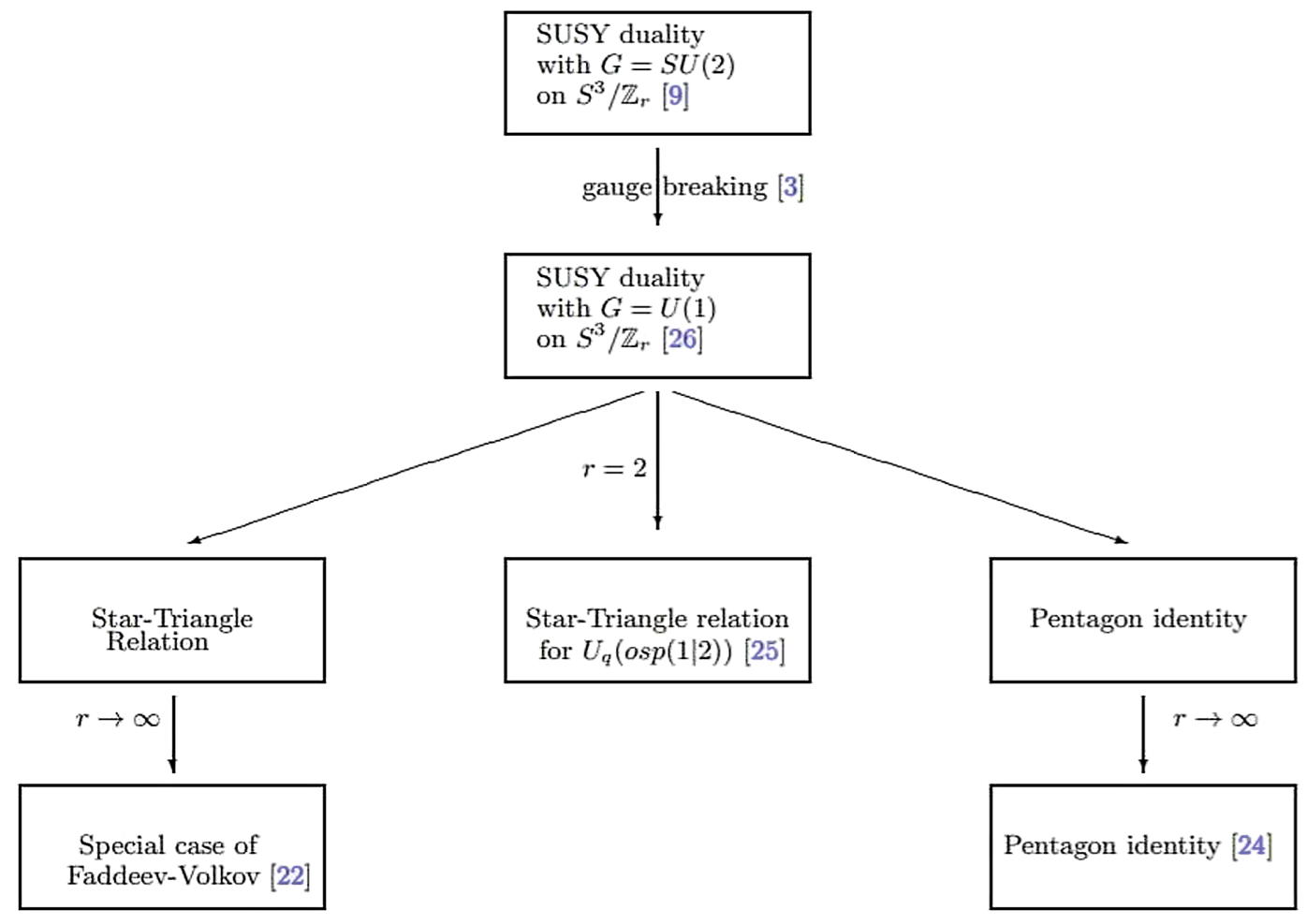

FIG. 1. Structure of the paper.

$$
\gamma^{(2)}\left(z ; \omega_{1}, \omega_{2}\right)=\exp \left(-\int_{0}^{\infty} \frac{d x}{x}\left[\frac{\sinh x\left(2 z-\omega_{1}-\omega_{2}\right)}{2 \sinh \left(x \omega_{1}\right) \sinh \left(x \omega_{2}\right)}-\frac{2 z-\omega_{1}-\omega_{2}}{2 x \omega_{1} \omega_{2}}\right]\right) .
$$

We obtain the identity (1.1) from the equality of partition functions of supersymmetric dual theories on $S^{3} / \mathbb{Z}_{r}$. The intriguing physical interpretation of this integral identity is that it can be written as the star-triangle relation for a certain two-dimensional Ising-type statistical model, as well as the pentagon identity for a certain triangulated 3-manifold. The integrable model based on the identity (1.1) is a generalization of the Faddeev-Volkov model [22,23] and a special case of the model can be found in [9]. Here we only construct the edge-interacting lattice spin model, however, the IRF version of the model may also give an interesting integral identity.

The Euler's gamma function limit of the integral identity (1.1) gives the known solution to the star-triangle relation [23] and also can be written as the pentagon identity presented in [24]. From the supersymmetric gauge theory side, by taking such a limit $(r \rightarrow \infty)$ one obtains the partition function of two-dimensional $\mathcal{N}=(2,2)$ supersymmetric gauge theories on two-sphere $S^{2}$.

A special case when $r=2$, the identity (1.1) gives the star-triangle relation discussed in [25] which was used for proving orthogonality and completeness relation of the
Clebsch-Gordan coefficients for the self-dual continuous series of $U_{q}(\operatorname{osp}(1 \mid 2))$. We expect an intimate relation between supersymmetric gauge theories on $S^{3} / \mathbb{Z}_{r}$, quantum groups $U_{q}(\operatorname{os} p(1 \mid 2))$ and two-dimensional conformal field theory.

Some results of the paper agree exactly with the work ${ }^{2}$ [26], based on a different interpretation of the integral identity (1.1). However our approach is based on the supersymmetric gauge theory computations.

The main idea of the paper is to construct connections between several solutions of the star-triangle equation and the pentagon relations. The Fig. 1 demonstrates the plan of the paper, pictorially.

The rest of this paper is organized as follows. In Sec. II, we briefly recollect some basic definitions. In Sec. III, we present the star-triangle relations and pentagon identities resulting from the supersymmetric duality. In Sec. IV, we discuss how to relate our star-triangle relation to the one

\footnotetext{
${ }^{2}$ The relation to the supersymmetric lens partition function was not discussed in [26].
} 
obtained in [25]. In Sec. V, we present our conclusions and discuss some open questions. We include three Appendixes for some technical details.

\section{LENS PARTITION FUNCTION, 3D DUALITY AND GAUGE SYMMETRY BREAKING}

\section{A. Supersymmetric partition function on $S_{b}^{\mathbf{3}} / \mathbb{Z}_{r}$}

We start by defining the general form of the three dimensional $\mathcal{N}=2$ partition function on the squashed lens space $S_{b}^{3} / \mathbb{Z}_{r}$. The lens partition function can be computed by a straightforward dimensional reduction of the four-dimensional lens superconformal index $[13,18,19]$ or via the supersymmetric localization technique $[14,15]$. Here we briefly outline some basic ingredients ${ }^{3}$ and refer the reader to $[9,14-16]$ for more details.

Recall that the lens space $S_{b}^{3} / \mathbb{Z}_{r}$ can be obtained from the squashed three sphere

$$
S_{b}^{3}=\left\{(x, y) \in \mathbb{C}^{2}, b^{2}|x|^{2}+b^{-2}|y|^{2}=1\right\}
$$

by making the identification $(x, y) \sim\left(e^{\frac{2 \pi i}{r}} x, e^{\frac{2 \pi i}{r}} y\right)$. The partition function on this manifold can be reduced to the following matrix model ${ }^{4}$

$Z=\sum_{m} \int \frac{1}{|W|} \prod_{j}^{r a n k G} \frac{d z_{j}}{2 \pi i r} Z_{\text {classical }}[z, m] Z_{\text {one-loop }}[z, m]$.

Here the sum is over the holonomies $m=\frac{r}{2 \pi} \int_{C} A_{\mu} d x^{\mu}$, where $C$ is the nontrivial cycle on $S_{b}^{3} / \mathbb{Z}_{r}$ and $A_{\mu}$ is the gauge field. The integral is over the Cartan subalgebra of the gauge group and $z_{j}$ variables are corresponding to Weyl weights. The order of the group $\mathrm{G}$ is represented by the prefactor $|W|$ such that the gauge group is broken by the holonomy into a product of $r$ subgroups.

The one-loop contribution of chiral multiplets is given in terms of hyperbolic hypergeometric function,

$$
\begin{aligned}
Z_{\text {chiral }}= & \prod_{j} \prod_{\rho_{j}} \prod_{\phi_{j}} \hat{s}_{b,-\rho_{j}(m)-\phi_{j}(n)} \\
& \times\left(i \frac{Q}{2}\left(1-\Delta_{j}\right)-\rho_{j}(z)-\phi_{j}(\Phi)\right) .
\end{aligned}
$$

\footnotetext{
${ }^{3}$ We mostly follow the notations of $[9,19]$.

${ }^{4}$ Actually, this expression is the Coulomb branch localization result, one can get the partition function in different forms depending on the chosen localization locus [27-29].
}

Here $j$ labels chiral multiplets; $\rho_{j}, \phi_{j}$, are the weights of the representation of the gauge and flavor groups, respectively and $\Delta_{j}$ is the Weyl weight of $j$ th chiral multiplet. We also define $Q=b+\frac{1}{b}$ with the squashing parameter $b^{2}=\omega_{2} / \omega_{1}$. The function $\hat{s}_{b, m}(z)$ is a version of the improved double sine function ${ }^{5}$ [9], which can be written as a product of hyperbolic gamma functions ${ }^{6}$

$$
\begin{aligned}
\hat{s}_{b,-m}(x)= & \sigma_{h}(m) \gamma^{(2)}\left(i z+y \omega_{1}+\eta ; \omega_{1} r, 2 \eta\right) \gamma^{(2)} \\
& \times\left(i z+\omega_{2}(r-y)+\eta ; \omega_{2} r, 2 \eta\right),
\end{aligned}
$$

with $\sigma_{h}(m)=e^{\frac{i \pi}{2 r} m(r-m)-(r-1) m^{2}}$ and $\eta=\left(\omega_{1}+\omega_{2}\right) / 2$. For practical reasons, and in keeping with supersymmetric gauge theories notations, we will mainly use the hyperbolic gamma function $\gamma^{(2)}\left(z, \omega_{1}, \omega_{2}\right)$ instead of $\hat{s}_{b, m}(z)$. The one-loop contribution of the vector multiplet combined with the Vandermonde determinant can be written as

$$
Z_{\mathrm{vector}}=\prod_{\alpha \in R_{+}} \frac{1}{\hat{s}_{b, \alpha(m)}\left(i \frac{Q}{2}+\alpha(z)\right)},
$$

where the product is over the positive roots $\alpha$ of the gauge group $G$. Once we know the group-theoretical data of three-dimensional supersymmetric theory on $S_{b}^{3} / \mathbb{Z}_{r}$, we can write down the partition function in terms of hyperbolic hypergeometric integral. Note that in our examples the classical term $Z_{\text {classical }}$ which includes the contributions coming from classical action of the Chern-Simons term and Fayet-Iliopoulos term will be absent. We should mention that the expressions for multiplets are the same as the one used in $[9,19]$ and differs by some factor (the partition function is the same) from that in $[14,16]$. The relation between two expressions can be found in [9] and in Appendix A.

\section{B. Three-dimensional $\mathcal{N}=2$ IR duality}

We will perform the gauge symmetry breaking of the following three-dimensional $\mathcal{N}=2$ supersymmetric dual theories ${ }^{7}$ :

\footnotetext{
${ }^{5}$ Let us mention that our $\hat{s}_{b}$ is different than one used in [30,31].

${ }^{6}$ Expressions in terms of the improved double sine function constitutes a special class of hyperbolic hypergeometric functions and they are in the interest of mathematical physics $[9,19,26,32,33]$.

${ }^{7}$ There is a four-dimensional version of this duality, see, e.g., $[34,35]$. The four-dimensional $\mathcal{N}=1$ theory also has $N_{f}=3$ flavors and usually dimensional reduction shifts the number of flavors by one. By adding a proper superpotential [36] one can obtain a duality with the same number of flavors as in four dimensions.
} 
(i) Theory A has gauge group ${ }^{8} S U(2)$ and flavor group $S U(6)$. The chiral multiples transform under the fundamental representation of the gauge group and the flavor group; the vector multiplet transforms under the adjoint representation of the gauge group.

(ii) Theory $\mathrm{B}$ is the dual to the theory $\mathrm{A}$ without gauge symmetry. There are 15 chiral multiplets in the totally antisymmetric tensor representation of the flavor group. In our case, theory B is the low energy description of theory A which can be characterized purely by composite gauge singlets.

Because of the supersymmetric duality, one obtains the following equality of the partition functions ${ }^{9}$ [9]

$$
\begin{aligned}
& \frac{1}{2 r \sqrt{-\omega_{1} \omega_{2}}} \sum_{y=0}^{[r / 2]} \epsilon(y) e^{\pi i C} \int_{-\infty}^{\infty} d z \frac{\prod_{i=1}^{6} \gamma^{(2)}\left(-i\left(a_{i} \pm z\right)-i \omega_{1}\left(u_{i} \pm y\right) ;-i \omega_{1} r,-i \omega_{1}-i \omega_{2}\right)}{\gamma^{(2)}\left(\mp 2 i z \mp 2 i \omega_{1} y ;-i \omega_{1} r,-i \omega_{1}-i \omega_{2}\right)} \\
& \quad \times \frac{\gamma^{(2)}\left(-i\left(a_{i} \pm z\right)-i \omega_{2}\left(r-\left(u_{i} \pm y\right)\right) ;-i \omega_{2} r,-i \omega_{1}-i \omega_{2}\right)}{\gamma^{(2)}\left(\mp 2 i z-i \omega_{2}(r \mp 2 y) ;-i \omega_{2} r,-i \omega_{1}-i \omega_{2}\right)} \\
&=\prod_{1 \leq i<j \leq 6} \gamma^{(2)}\left(-i\left(a_{i}+a_{j}\right)-i \omega_{1}\left(u_{i}+u_{j}\right) ;-i \omega_{1} r,-i \omega_{1}-i \omega_{2}\right) \\
& \gamma^{(2)}\left(-i\left(a_{i}+a_{j}\right)-i \omega_{2}\left(r-\left(u_{i}+u_{j}\right)\right) ;-i \omega_{2} r,-i \omega_{1}-i \omega_{2}\right),
\end{aligned}
$$

with the balancing condition $i \sum_{i=1}^{6} a_{i}=\omega_{1}+\omega_{2}$ and $\sum_{i=1}^{6} u_{i}=0$, where $C=2 y^{2}-\sum_{i=1}^{6} u_{i}^{2}$. We should mention that there is a contribution of the $R$-symmetry appearing in the partition function but we absorbed it in the flavor fugacity. Since all physical degrees of freedom of theory B are gauge invariant there is no summation and integration on the right-hand side of the identity. The case $r=1$ of the integral identity (2.5) is a very well-known integral identity, see, e.g., [30]; in this case it corresponds to the equality of the squashed threesphere partition functions [39].

The hyperbolic hypergeometric beta sum-integral (2.5) is an important identity in the theory of hyperbolic hypergeometric functions. Its role in integrable models of statistical mechanics was discovered in [9].

\section{Gauge symmetry breaking}

Now we are in a position to obtain new dual theories by breaking the gauge symmetry. The idea is to break the

\footnotetext{
${ }^{8}$ Actually this duality is a special case of the family of dualities for the gauge group $\operatorname{SP}\left(2 N_{c}\right)$. For $N_{c}=1$ this duality coincides with the $S U(2)[37,38]$.

${ }^{9} \mathrm{We}$ will not go into details of the evaluation of partition functions for these dual theories, see [9] and references therein.
}

gauge symmetry from $S U(2)$ to $U(1)$ in dual theories presented above. We give a VEV to two flavor quarks, breaking the gauge group to $U(1)$ and reducing the flavor group to $S U(3) \times S U(3)$. As a result we obtain the following dual theories:

(i) Theory $\mathrm{A}: 3 \mathrm{~d} \mathcal{N}=2$ theory with $U(1)$ gauge symmetry and $S U(3)_{L} \times S U(3)_{R}$ flavor group, chiral multiplets belong to the $S U(3)_{L}$ transforming in the fundamental representation of the gauge group and chiral multiplets belong to the $S U(3)_{R}$, transforming in the antifundamental representation.

(ii) Theory B: the dual theory has the same global symmetries without gauge degrees of freedom, nine "mesons," transforming in the fundamental representation of the flavor group $S U(3)_{L} \times$ $S U(3)_{R}$.

We make the breaking of gauge symmetry on the level of partition functions. Following the work [3] (see also [26]) let us replace the flavor fugacities ${ }^{10} a_{i}$ to $a_{i}+\mu$ for $i=\{1,2,3\}$ and $a_{i}-\mu$ for $i=\{4,5,6\}$. We use the fact that the identity (2.5) is symmetric with respect to $z \rightarrow-z$ transformation. By changing the variable $z$ to $z+\mu$ we get the following expression:

\footnotetext{
${ }^{10}$ In three dimensions it is a complexified real mass parameter.
} 


$$
\begin{aligned}
\frac{1}{r \sqrt{-\omega_{1} \omega_{2}}} \sum_{y=0}^{[r / 2]} e^{\frac{\pi i c}{2}} e(y) \int_{-\mu}^{\infty} d z \frac{\prod_{i=1}^{3} \gamma^{(2)}\left(-i\left(a_{i}+\mu \pm(z+\mu)\right)-i \omega_{1}\left(u_{i} \pm y\right) ;-i \omega_{1} r,-i \omega_{1}-i \omega_{2}\right)}{\gamma^{(2)}\left(\mp 2 i(z+\mu) \mp 2 i \omega_{1} y ;-i \omega_{1} r,-i \omega_{1}-i \omega_{2}\right)} \\
\quad \times \frac{\gamma^{(2)}\left(-i\left(a_{i}+\mu \pm(z+\mu)\right)-i \omega_{2}\left(r-\left(u_{i} \pm y\right)\right) ;-i \omega_{2} r,-i \omega_{1}-i \omega_{2}\right)}{\gamma^{(2)}\left(\mp 2 i(z+\mu)-i \omega_{2}(r \mp 2 y) ;-i \omega_{2} r,-i \omega_{1}-i \omega_{2}\right)} \\
\quad \times \prod_{i=4}^{6} \gamma^{(2)}\left(-i\left(a_{i}-\mu \pm(z+\mu)\right)-i \omega_{1}\left(u_{i} \pm y\right) ;-i \omega_{1} r,-i \omega_{1}-i \omega_{2}\right) \\
\quad \times \gamma^{(2)}\left(-i\left(a_{i}-\mu \pm(z+\mu)\right)-i \omega_{2}\left(r-\left(u_{i} \pm y\right)\right) ;-i \omega_{2} r,-i \omega_{1}-i \omega_{2}\right) \\
=\prod_{i=1}^{3} \prod_{j=4}^{6} \gamma^{(2)}\left(-i\left(a_{i}+a_{j}\right)-i \omega_{1}\left(u_{i}+u_{j}\right) ;-i \omega_{1} r,-i \omega_{1}-i \omega_{2}\right) \gamma^{(2)}\left(-i\left(a_{i}+a_{j}\right)-i \omega_{2}\left(r-\left(u_{i}+u_{j}\right)\right) ;-i \omega_{2} r,-i \omega_{1}+\omega_{2}\right) \\
\quad \times \prod_{1 \leq i<j \leq 3} \gamma^{(2)}\left(-i\left(a_{i+3}+a_{j+3}-2 \mu\right)-i \omega_{2}\left(r-\left(u_{i+3}+u_{j+3}\right)\right) ;-i \omega_{2} r,-i \omega_{1}-i \omega_{2}\right) \\
\quad \times \gamma^{(2)}\left(-i\left(a_{i}+a_{j}+2 \mu\right)-i \omega_{2}\left(r-\left(u_{i}+u_{j}\right)\right) ;-i \omega_{2} r,-i \omega_{1}-i \omega_{2}\right) \\
\quad \times \gamma^{(2)}\left(-i\left(a_{i+3}+a_{j+3}-2 \mu\right)-i \omega_{1}\left(u_{i+3}+u_{j+3}\right) ;-i \omega_{1} r,-i \omega_{1}-i \omega_{2}\right) \\
\quad \times \gamma^{(2)}\left(-i\left(a_{i}+a_{j}+2 \mu\right)-i \omega_{1}\left(u_{i}+u_{j}\right) ;-i \omega_{1} r,-i \omega_{1}-i \omega_{2}\right)
\end{aligned}
$$

where $C=2 y^{2}-\sum_{i=1}^{6} u_{i}^{2}$. After taking the limit $\mu \rightarrow \infty$ and renaming the flavor group coefficients as $a_{i}=b_{i}$ and $u_{i}=v_{i}$ for only $i \in\{4,5,6\}$, the reduced form of the hyperbolic hypergeometric integral identity turns out to be

$$
\begin{aligned}
& \frac{1}{r \sqrt{-\omega_{1} \omega_{2}}} \sum_{y=0}^{r r / 2]} \epsilon(y) e^{\frac{\pi i c}{2}} \int_{-\infty}^{\infty} d z \prod_{i=1}^{3} \gamma^{(2)}\left(-i\left(a_{i}-z\right)-i \omega_{1}\left(u_{i}-y\right) ;-i \omega_{1} r,-i \omega_{1}-i \omega_{2}\right) \\
& \times \gamma^{(2)}\left(-i\left(a_{i}-z\right)-i \omega_{2}\left(r-\left(u_{i}-y\right)\right) ;-i \omega_{2} r,-i \omega_{1}-i \omega_{2}\right) \gamma^{(2)}\left(-i\left(b_{i}+z\right)-i \omega_{1}\left(v_{i}+y\right) ;-i \omega_{1} r,-i \omega_{1}-i \omega_{2}\right) \\
& \times \gamma^{(2)}\left(-i\left(b_{i}+z\right)-i \omega_{2}\left(r-\left(v_{i}+y\right)\right) ;-i \omega_{2} r,-i \omega_{1}-i \omega_{2}\right) \\
& \left.=\prod_{i, j=1}^{3} \gamma^{(2)}\left(-i\left(a_{i}+b_{j}\right)-i \omega_{1}\left(u_{i}+v_{j}\right) ;-i \omega_{1} r,-i \omega_{1}-i \omega_{2}\right) \gamma^{(2)}\left(-i\left(a_{i}+b_{j}\right)-i \omega_{2}\left(r-\left(u_{i}+v_{j}\right)\right) ;-i \omega_{2} r,-i \omega_{1}-i \omega_{2}\right)\right),
\end{aligned}
$$

with the balancing conditions $\sum_{i} a_{i}+b_{i}=\omega_{1}+\omega_{2}$ and $\sum_{i} u_{i}+v_{i}=0$. Here $C=-2 y+\left(u_{1}+u_{2}+u_{3}-v_{1}-\right.$ $\left.v_{2}-v_{3}\right)$. On the left-hand side of the integral identity, we see the partition function of theory A and on the right-hand side, theory B. A similar identity was discussed for the $S^{3}$ sphere partition functions in $[3,40]$ and for the superconformal indices in [41-43]. The integral identity (2.7) is essentially the same integral identity as the one obtained in [26] with a slightly different sign coefficient.

\section{STAR-TRIANGLE RELATION AND PENTAGON IDENTITY}

In this section we investigate the relation between supersymmetric dualities, integrability, and triangulated 3-manifolds. We will show that the integral identity (2.7) can be written as the star-triangle relation and as the integral pentagon identity.

\section{A. Pentagon identity}

The integral identity (2.7) can be written as a pentagon relation. The pentagon identity usually represents the basic 2-3 Pachner move [44] for a certain triangulated 3-manifold. There are several examples of integral pentagon relations computed via three-dimensional supersymmetric dualities, see, e.g., [24,40-43,45-47]. Here we present a new pentagon identity in terms of hyperbolic gamma functions.

It is convenient to define the following function:

$$
\begin{aligned}
\mathcal{B}\left(z_{1}, u_{1} ; z_{2}, u_{2}\right)= & \frac{\left.\gamma^{(2)}\left(-i z_{1}-i \omega_{1} u_{1} ;-i \omega_{1},-i \omega_{1}-i \omega_{2}\right) \gamma^{(2)}\left(-i z_{1}-i \omega_{2}\left(r-u_{1}\right) ;-i \omega_{2},-i \omega_{1}-i \omega_{2}\right)\right)}{\left.\gamma^{(2)}\left(-i\left(z_{1}+z_{2}\right)-i \omega_{1}\left(u_{1}+u_{2}\right) ;-i \omega_{1},-i \omega_{1}-i \omega_{2}\right)\right)} \\
& \times \frac{\gamma^{(2)}\left(-i z_{2}-i \omega_{1} u_{2} ;-i \omega_{1},-i \omega_{1}-i \omega_{2}\right) \gamma^{(2)}\left(-i z_{2}-i \omega_{2}\left(r-u_{2}\right) ;-i \omega_{2},-i \omega_{1}-i \omega_{2}\right)}{\gamma^{(2)}\left(-i\left(z_{1}+z_{2}\right)-i \omega_{2}\left(r-u_{1}-u_{2}\right) ;-i \omega_{2},-i \omega_{1}-i \omega_{2}\right)},
\end{aligned}
$$


which solves the following integral pentagon identity, ${ }^{11}$

$$
\begin{aligned}
& \frac{1}{r \sqrt{-\omega_{1} \omega_{2}}} \sum_{y=-\lfloor r / 2\rfloor}^{\lfloor r / 2\rfloor} e^{\frac{\pi i C}{2}} \int_{-\infty}^{\infty} d z \prod_{i=1}^{3} \mathcal{B}\left(a_{i}-z, u_{i}-y ; b_{i}+z, v_{i}+y\right) \\
& \quad=\mathcal{B}\left(a_{1}+b_{2}, u_{1}+v_{2} ; a_{2}+b_{3}, u_{2}+v_{3}\right) \mathcal{B}\left(a_{1}+b_{3}, u_{1}+v_{3} ; a_{2}+b_{1}, u_{2}+v_{1}\right)
\end{aligned}
$$

with the same balancing conditions given in (2.7).

\section{B. Limit of the pentagon identity}

There are several pentagon identities in terms of Euler's gamma function [24,40,49]. Here we present the pentagon relation found in [24] in terms of the gamma function which we obtain in a different manner. ${ }^{12}$

In order to explore the limit of the pentagon identity we use the following asymptotic relation:

$$
\lim _{\omega_{2} \rightarrow \infty}\left(\frac{\omega_{2}}{2 \pi \omega_{1}}\right)^{\frac{z}{\omega_{2}}-\frac{1}{2}} \gamma^{(2)}\left(z ; \omega_{1}, \omega_{2}\right)=\frac{\Gamma\left(z / \omega_{1}\right)}{\sqrt{2 \pi}} .
$$

We identify $\omega_{1}$ with $\omega_{2}$ and redefine all coefficients dividing by $\frac{\omega_{1}+\omega_{2}}{\omega_{1}}$, than by altering $\frac{a_{i}-z}{\omega_{1}}$ to $a_{i}-z$, we obtain the limit of the pentagon identity as follows:

$$
\begin{aligned}
& \sum_{y=-\infty}^{\infty} \int_{-\infty}^{\infty} \frac{d z}{4 \pi i} \prod_{i=1}^{3} \frac{\Gamma\left(a_{i}-z+\frac{u_{i}-y}{2}\right) \Gamma\left(b_{i}+z+\frac{v_{i}+y}{2}\right) \Gamma\left(1-a_{i}-b_{i}+\frac{u_{i}+v_{i}}{2}\right)}{\Gamma\left(1-b_{i}-z+\frac{v_{i}+y}{2}\right) \Gamma\left(1-a_{i}+z+\frac{u_{i}-y}{2}\right) \Gamma\left(a_{i}+b_{i}+\frac{u_{i}+v_{i}}{2}\right)} \\
& \quad=\prod_{i, j=1 ; i \neq j}^{3} \frac{\Gamma\left(a_{i}+b_{j}+\frac{u_{i}+v_{j}}{2}\right)}{\Gamma\left(1-a_{i}-b_{j}+\frac{u_{i}+v_{j}}{2}\right)} .
\end{aligned}
$$

If we introduce the following function

$$
\mathcal{B}\left(z_{1}, u_{1} ; z_{2}, u_{2}\right)=\frac{\Gamma\left(z_{1}+\frac{u_{1}}{2}\right) \Gamma\left(z_{2}+\frac{u_{2}}{2}\right) \Gamma\left(1-z_{1}-z_{2}-\frac{u_{1}+u_{2}}{2}\right)}{\Gamma\left(z_{1}+z_{2}+\frac{u_{1}+u_{2}}{2}\right) \Gamma\left(1-z_{1}-\frac{u_{1}}{2}\right) \Gamma\left(1-z_{2}-\frac{u_{2}}{2}\right)},
$$

one obtains the integral pentagon identity

$$
\begin{aligned}
& \sum_{y=-\infty}^{\infty} \int_{-\infty}^{\infty} \frac{d z}{4 \pi i} \prod_{i=1}^{3} \mathcal{B}\left(a_{i}-z, u_{i}-y ; b_{i}+z, v_{i}+y\right) \\
& \quad=\mathcal{B}\left(a_{1}+b_{3}, u_{1}+v_{3} ; a_{2}+b_{1}, u_{2}+v_{1}\right) \mathcal{B}\left(a_{1}+b_{2}, u_{1}+v_{2} ; a_{2}+b_{3}, u_{1}+v_{3}\right) .
\end{aligned}
$$

This is exactly the result obtained in [24] via dimensional reduction of the three-dimensional $\mathcal{N}=2$ supersymmetric dual theories on $S^{2} \times S^{1}$.

\section{Star-triangle relation}

The star-triangle relation is a crucial equation in the study of two-dimensional integrable lattice spin models. Here we obtain the solution to the star-triangle relation mentioned in [26]. We fix the parameters as

$$
a_{i}=-\alpha_{i}+x_{i}, \quad b_{i}=-\alpha_{i}-x_{i},
$$

and we insert the condition $u_{i}=-v_{i}$. By defining the Boltzmann weight as

\footnotetext{
${ }^{11}$ One can think that our pentagon relation coincides with the one obtained in [48]. However they are different; actually the identity (3.10) [or (4.12)] in [48] can be obtained from the three-dimensional $\mathcal{N}=2$ mirror symmetry on $S^{3} / \mathbb{Z}_{k}$ for special values of flavor fugacities, see [14].

${ }^{72}$ The derivation of the pentagon identity in [24] is based on the reduction procedure of the three-dimensional $\mathcal{N}=2$ superconformal index (the partition function on $S^{2} \times S^{1}$ ) to the $\mathcal{N}=(2,2)$ supersymmetric sphere partition function by shrinking the radius of $S^{1}$ [50].
} 


$$
\begin{aligned}
W_{\alpha}\left(x_{i}, x_{j}, u_{i}, u_{j}\right)= & e^{-\pi i\left(u_{i}+u_{j}\right)} \gamma^{(2)}\left(-i\left(-\alpha+x_{i}-x_{j}\right)-i \omega_{1}\left(u_{i}-u_{j}\right) ;-i \omega_{1},-i\left(\omega_{1}+\omega_{2}\right)\right) \\
& \times \gamma^{(2)}\left(-i\left(-\alpha+x_{i}-x_{j}\right)-i \omega_{2}\left(r-\left(u_{i}-u_{j}\right)\right) ;-i \omega_{2},-i\left(\omega_{1}+\omega_{2}\right)\right) \\
& \times \gamma^{(2)}\left(-i\left(-\alpha-x_{i}+x_{j}\right)-i \omega_{1}\left(u_{j}-u_{i}\right) ;-i \omega_{1},-i\left(\omega_{1}+\omega_{2}\right)\right) \\
& \times \gamma^{(2)}\left(-i\left(-\alpha-x_{i}+x_{j}\right)-i \omega_{2}\left(r-\left(u_{j}-u_{i}\right)\right) ;-i \omega_{2},-i\left(\omega_{1}+\omega_{2}\right)\right)
\end{aligned}
$$

and the spin-independent weight as

$$
R\left(\alpha_{1}, \alpha_{2}, \alpha_{3}\right)=\prod_{j=1}^{3} \gamma^{(2)}\left(2 i \alpha_{j} ;-i \omega_{1},-i\left(\omega_{1}+\omega_{2}\right)\right) \gamma^{(2)}\left(2 i \alpha_{j} ;-i \omega_{2},-i\left(\omega_{1}+\omega_{2}\right)\right),
$$

one can rewrite the integral identity (2.7) as the following star-triangle relation:

$$
\begin{aligned}
& \frac{1}{r \sqrt{-\omega_{1} \omega_{2}}} \sum_{y=-\lfloor r / 2\rfloor}^{\lfloor r / 2\rfloor} e^{-2 \pi i y} \int_{-\infty}^{\infty} d z W_{\alpha_{1}}\left(x_{1}, z, u_{1}, y\right) W_{\alpha_{2}}\left(x_{2}, z, u_{2}, y\right) W_{\alpha_{3}}\left(x_{3}, z, u_{3}, y\right) \\
& =R\left(\alpha_{1}, \alpha_{2}, \alpha_{3}\right) W_{\alpha_{1}+\alpha_{2}}\left(x_{1}, x_{2}, u_{1}, u_{2}\right) W_{\alpha_{1}+\alpha_{3}}\left(x_{1}, x_{3}, u_{1}, u_{3}\right) W_{\alpha_{2}+\alpha_{3}}\left(x_{2}, x_{3}, u_{2}, u_{3}\right) .
\end{aligned}
$$

Our model is an Ising type model where sites of the lattice are assigned to discrete $u$ and continuous spin $x$ variables.

\section{Limit of the star-triangle relation}

There are several solutions to the star-triangle relation in terms of Euler's gamma function. In our case such a solution can be achieved by taking the limit (3.3). After taking the limit, we obtain the following Boltzmann weight

$$
W_{\theta}(x, z, u, y)=\frac{\Gamma\left(\frac{1-\frac{\theta}{\pi}+i x-i z+u-y}{2}\right) \Gamma\left(\frac{1-\frac{\theta}{\pi}-i x+i z+v+y}{2}\right)}{\Gamma\left(\frac{1+\frac{\theta}{\pi}+i x-i z-u+y}{2}\right) \Gamma\left(\frac{1+\frac{\theta}{\pi}-i x+i z-u+y}{2}\right)},
$$

and spin independent weight

$$
R\left(\theta_{1}, \theta_{2}, \theta_{3}\right)=\prod_{i=1}^{3} \frac{\Gamma\left(1-\frac{\theta_{i}}{\pi}\right)}{\Gamma\left(\frac{\theta_{i}}{\pi}\right)} .
$$

The star-triangle relation can be written as

$$
\begin{aligned}
& \sum_{y=-\infty}^{\infty} \int_{-\infty}^{\infty} \frac{d z}{8 \pi} W_{\theta_{1}}\left(x_{1}, z, u_{1}, y\right) W_{\theta_{2}}\left(x_{2}, z, u_{2}, y\right) W_{\theta_{3}}\left(x_{3}, z, u_{3}, y\right) \\
& \quad=R\left(\theta_{1}, \theta_{2}, \theta_{3}\right) W_{\pi-\theta_{1}}\left(x_{2}, x_{3}, u_{2}, u_{3}\right) W_{\pi-\theta_{2}}\left(x_{1}, x_{3}, u_{1}, u_{3}\right) W_{\pi-\theta_{3}}\left(x_{1}, x_{2}, u_{1}, u_{2}\right),
\end{aligned}
$$

where $\theta_{1}+\theta_{2}+\theta_{3}=2 \pi$. It can be easily checked that this solution is exactly the one obtained in [23] from the FaddeevVolkov model. In [23] the authors normalized the Boltmann weights (3.11) in such a way that the spin-independent function $R\left(\theta_{1}, \theta_{2}, \theta_{3}\right)$ is equal to one. Note that the solution (3.11) is related to the special case of the Zamolodchikov's "fishnet" model $[23,51,52]$.

\section{RELATION TO THE $U_{q}(\operatorname{osp}(1 \mid 2))$}

It is well known that the unitary representations of the modular double of $U_{q}(\operatorname{sl}(2, R))$ is equivalent to the representations of the Liouville theory. For instance, $3 j$ symbols for the tensor product of modular double representations of $U_{q}(\operatorname{sl}(2, R))$ appear in the fusion product for the Liouville vertex operators. The modular double representation for the $U_{q}(\operatorname{osp}(1 \mid 2))$ plays $^{13}$ the same role in the $\mathcal{N}=1$ supersymmetric Liouville theory.

\footnotetext{
${ }^{13}$ It is the $q$-deformed universal enveloping algebra of the Lie superalgebra $\operatorname{ss} p(1 \mid 2)$ with the deformation parameter $q=e^{i \pi b^{2}}[53-55]$.
} 
Here we show how one can obtain the star-triangle relation for the $U_{q}(\operatorname{osp}(1 \mid 2))[25,56]$ (see also [57]) from the integral identity (2.7). The computations presented here and in Appendix C overlap with the computations of [26]. We use different notations and present all calculations in detail, see Appendix C. From the supersymmetric gauge theory point of view, the star-triangle relation represents the equality of partition functions of dual three-dimensional $\mathcal{N}=2$ gauge theories on $S^{3} / Z_{2}$ (it is topologically $\mathbb{R} \mathbb{P}^{3}$ ). Similar computations for the Liouville field theory and the supersymmetric gauge theories on squashed three-sphere $S_{b}^{3}$ was performed in [58].

A special case of the expression (2.7) when $r=2$, gives the star-triangle relation discussed in [25] which can be used for proving the orthogonality and completeness relation of the Clebsch-Gordan coefficients for the selfdual continuous series of $U_{q}(\operatorname{osp}(1 \mid 2))$ and the $\mathcal{N}=1$ supersymmetric Liouville theory. For the special case $r=2$ we obtain the following expression ${ }^{14}$

$$
\begin{aligned}
& \sum_{\nu=0,1}(-1)^{\frac{2 \nu-3-\sum_{i}\left(\mu_{i}-\nu_{i}\right)}{2}} \int \frac{d x}{i} \prod_{i=1}^{3} S_{\nu+\nu_{i}}\left(x+a_{i}\right) S_{1+\nu+\mu_{i}}\left(b_{i}-x\right) \\
& \quad=2 \prod_{i, j=1}^{3} S_{1+\nu_{i}+\mu_{j}}\left(a_{i}+b_{j}\right)
\end{aligned}
$$

where we introduced the notations of the work [25]

$S_{\nu}(x)=\gamma^{(2)}\left(\frac{x+(1-\nu) b}{2}, b, \frac{1}{b}\right) \gamma^{(2)}\left(\frac{x+1 / b+\nu b}{2}, b, \frac{1}{b}\right)$.

Note that we have a different sign coefficient ${ }^{15}$ in (4.1) than in $[25,26]$. It seems that one can obtain the integral identity (5.14) from the work [59] by tending one of the flavor fugacities to $b+\frac{1}{b}$.

\section{CONCLUSION}

We obtain the pentagon identity (3.2) (related to the Heisenberg double) and the star-triangle relation (3.10) (related to the quantum algebra) from the same integral identity. Note that it is possible to construct the Boltzmann weight $W$ (3.8) from the $B$-function (3.1), see e.g., [60-62].

\footnotetext{
${ }^{14}$ For details, see Appendix C.

${ }^{15}$ In [59], the identity is given in the form

$$
\begin{aligned}
& \sum_{\nu=0,1}(-1)^{\nu\left(1+\sum_{i}\left(\nu_{i}+\mu_{i}\right)\right) / 2} \int \frac{d x}{i} \prod_{i=1}^{3} S_{\nu+\nu_{i}}\left(x+a_{i}\right) S_{1+\nu+\mu_{i}}\left(b_{i}-x\right) \\
& \quad=2 \prod_{i, j=1}^{3} S_{1+\nu_{i}+\mu_{j}}\left(a_{i}+b_{j}\right) .
\end{aligned}
$$
}

There are several directions that we wish to pursue in the future. We showed that, by performing a suitable identification, our star-triangle relation gives the same identity obtained in [25]. This result is interesting not only because it builds a relation between two different subjects, but also it can be applied to arbitrary $r$. The problem we leave to the future is the construction of the corresponding quantum algebra for the integral identity (3.13) with the general $r$.

In this work we presented rational and trigonometric solutions to the Yang-Baxter equation in the form called star-triangle relation. It would be interesting to construct the operator form of the Yang-Baxter equation and the Hamiltonian of the one-dimensional chain corresponding to this solution.

The pentagon identity and the star-triangle relation are a consequence of the Heisenberg double and the quantum algebra, respectively. We should mention that the appearance of the pentagon relation refers to the Pachner's move for triangulated 3-manifolds, though, we do not know how to construct this relation formally.

\section{ACKNOWLEDGMENTS}

It is a pleasure to acknowledge Ege Eren and Shahriyar Jafarzade for our collaboration at an early stage of this work. We would like to thank Michal Pawelkiewicz for sharing his notes and his thesis. The work of I. G. is partially supported by the Bogazici University Research Fund under Grant No. 20B03SUP3 and by the BAP Project (No. 2019-26) funded by Mimar Sinan Fine Arts University. M. M. is supported by the 2209-TUBITAK National/International Research Projects Fellowship Program for Undergraduate Students under Grant No. 1919B011902237.

\section{APPENDIX A: PROPERTIES OF SPECIAL FUNCTIONS}

Here we present several definitions and notations of special functions needed in this work.

We briefly summarize the basic properties of hyperbolic gamma function and its different notations [30,63]. This function appears in several areas of mathematical and theoretical physics; here is an incomplete list of these topics:

(i) knot theory [62,64-66],

(ii) supersymmetric gauge theory [58],

(iii) integrable models of statistical mechanics $[22,23,67]$,

(iv) special functions [30].

With parameters $\tilde{q}=e^{2 \pi i \omega_{1} / \omega_{2}}$ and $q=e^{-2 \pi i \omega_{2} / \omega_{1}}$, the infinite product representation is

$$
\gamma^{(2)}\left(z ; \omega_{1}, \omega_{2}\right)=e^{-\frac{\pi i}{2} B_{2,2}\left(z ; \omega_{1}, \omega_{2}\right)} \frac{\left(e^{2 \pi i \frac{z}{\omega_{2}}} \tilde{q} ; \tilde{q}\right)}{\left(e^{2 \pi i \frac{z}{\omega_{1}}} ; q\right)},
$$

where we have one of the Bernoulli polynomials, 


$$
B_{2,2}\left(z ; \omega_{1}, \omega_{2}\right)=\frac{z^{2}-z\left(\omega_{1}+\omega_{2}\right)}{\omega_{1} \omega_{2}}+\frac{\omega_{1}^{2}+3 \omega_{1} \omega_{2}+\omega_{2}^{2}}{6 \omega_{1} \omega_{2}}
$$

Here, we realize that $B_{2,2}\left(z ; \omega_{1}, \omega_{2}\right)$ is crucial for the asymptotic behavior of the hyperbolic gamma function. The hyperbolic gamma function has an integral representation ${ }^{16}$

$$
\gamma^{(2)}\left(z ; \omega_{1}, \omega_{2}\right)=\exp \left(-\int_{0}^{\infty} \frac{d x}{x}\left[\frac{\sinh x\left(2 z-\omega_{1}-\omega_{2}\right)}{2 \sinh \left(x \omega_{1}\right) \sinh \left(x \omega_{2}\right)}-\frac{2 z-\omega_{1}-\omega_{2}}{2 x \omega_{1} \omega_{2}}\right]\right),
$$

where $\operatorname{Re}\left(\omega_{1}\right), \operatorname{Re}\left(\omega_{2}\right)>0$ and $\operatorname{Re}\left(\omega_{1}+\omega_{2}\right)>\operatorname{Re}(z)>0$. We list here some properties of this function:

$$
\begin{gathered}
\text { symmetry: } \quad \gamma^{(2)}\left(z ; \omega_{1}, \omega_{2}\right)=\gamma^{(2)}\left(z ; \omega_{2}, \omega_{1}\right), \\
\text { reflection: } \quad \gamma^{(2)}\left(z ; \omega_{1}, \omega_{2}\right) \gamma^{(2)}\left(\omega_{1}+\omega_{2}-z ; \omega_{1}, \omega_{2}\right)=1, \\
\text { scaling: } \quad \gamma^{(2)}\left(z ; \omega_{1}, \omega_{2}\right)=\gamma^{(2)}\left(u z ; u \omega_{1}, u \omega_{2}\right), \\
\text { conjugation: } \quad \gamma^{(2)}\left(z ; \omega_{1}, \omega_{2}\right)^{*}=\gamma^{(2)}\left(z^{*} ; \omega_{2}^{*}, \omega_{1}^{*}\right) .
\end{gathered}
$$

Another very important property of the hyperbolic gamma function is the following difference equation:

$$
\gamma^{(2)}\left(z+\omega_{1} ; \omega_{1}, \omega_{2}\right)=e^{-\frac{\pi i}{2}\left(B_{2,2}\left(z+\omega_{1} ; \omega\right)-B_{2,2}(z ; \omega)\right)}\left(1-e^{2 \pi i \frac{z}{\omega_{2}}}\right) \gamma^{(2)}\left(z ; \omega_{1}, \omega_{2}\right),
$$

after simplifying, the difference equation takes the form,

$$
\gamma^{(2)}\left(z+\omega_{1} ; \omega_{1}, \omega_{2}\right)=2 \sin \left(\frac{\pi z}{\omega_{2}}\right) \gamma^{(2)}\left(z ; \omega_{1}, \omega_{2}\right)
$$

Now we introduce the asymptotic behavior of the function

$$
\begin{array}{ll}
\lim _{z \rightarrow \infty} e^{\frac{\pi i}{2} B_{2,2}\left(z ; \omega_{1}, \omega_{2}\right)} \gamma^{(2)}\left(z ; \omega_{1}, \omega_{2}\right)=1 & \text { for } \arg \omega_{2}+\pi>\arg z>\arg \omega_{1}, \\
\lim _{z \rightarrow \infty} e^{-\frac{\pi i}{2} B_{2,2}\left(z ; \omega_{1}, \omega_{2}\right)} \gamma^{(2)}\left(z ; \omega_{1}, \omega_{2}\right)=1 & \text { for } \arg \omega_{2}>\arg z>\arg \omega_{1}-\pi,
\end{array}
$$

where $\operatorname{Im}\left(\frac{\omega_{1}}{\omega_{2}}\right)>0$. We use these formulas for the breaking of gauge symmetry given in Appendix B.

There is a generalization of the hyperbolic gamma function $\Gamma_{h}\left(z, y ; \omega_{1}, \omega_{2}\right)$ which was introduced in [9]. This function can be defined in terms of $\gamma^{(2)}\left(z ; \omega_{1}, \omega_{2}\right)$ as follows:

$$
\Gamma_{h}\left(z, m ; \omega_{1}, \omega_{2}\right)=e^{\phi(m)} \gamma^{(2)}\left(-i z-i \omega_{1} m ;-i \omega_{1} r,-2 i \eta\right) \gamma^{(2)}\left(-i z-i \omega_{2}(r-m) ;-i \omega_{2} r,-2 i \eta\right),
$$

where $\phi(m)=-\frac{\pi i}{6 r}\left(2 m^{3}-3 m^{2} r+y r^{2}\right)$. It has the following properties:

$$
\begin{aligned}
\text { symmetry and reflection: } & \Gamma_{h}\left(z, y ; \omega_{1}, \omega_{2}\right) \Gamma_{h}\left(\omega_{1}+\omega_{2}-z, y ; \omega_{2}, \omega_{1}\right)=e^{2 \phi(y)}, \\
\text { scaling: } & \frac{\Gamma_{h}\left(u z, u y ; u \omega_{1}, u \omega_{2}\right)}{\Gamma_{h}\left(z, y ; \omega_{2}, \omega_{1}\right)}=e^{\phi(u y)-\phi(y)}, \\
\text { conjugation: } & \Gamma_{h}\left(z, y ; \omega_{1}, \omega_{2}\right)^{*}=\Gamma_{h}\left(z^{*}, y^{*} ; \omega_{2}^{*}, \omega_{1}^{*}\right) .
\end{aligned}
$$

\footnotetext{
${ }^{16}$ Actually, there are several integral representations, see, e.g., $[68,69]$.
} 


\section{APPENDIX B: GAUGE SYMMETRY BREAKING}

We start by reparemetrizing the integral identity coming from the duality argument, given in (2.7)

$$
\begin{aligned}
& \frac{1}{2 r \sqrt{-\omega_{1} \omega_{2}}} \sum_{y=0}^{[r / 2]} \epsilon(y) e^{\pi i C} \int_{-\infty}^{\infty} d z \frac{\prod_{i=1}^{6} \gamma^{(2)}\left(-i\left(a_{i} \pm z\right)-i \omega_{1}\left(u_{i} \pm y\right) ;-i \omega_{1} r,-i\left(\omega_{1}+\omega_{2}\right)\right)}{\gamma^{(2)}\left(\mp 2 i z \mp i \omega_{1}(2 y) ;-i \omega_{1} r,-i\left(\omega_{1}+\omega_{2}\right)\right)} \\
& \quad \times \frac{\gamma^{(2)}\left(-i\left(a_{i} \pm z\right)-i \omega_{2}\left(r-\left(u_{i} \pm y\right)\right) ;-i \omega_{2} r,-i\left(\omega_{1}+\omega_{2}\right)\right)}{\gamma^{(2)}\left(\mp 2 i z-i \omega_{2}(r \mp(2 y)) ;-i \omega_{2} r,-i\left(\omega_{1}+\omega_{2}\right)\right)} \\
& =\prod_{1 \leq i<j \leq 6} \gamma^{(2)}\left(-i\left(a_{i}+a_{j}\right)-i \omega_{1}\left(u_{i}+u_{j}\right) ;-i \omega_{1} r,-i\left(\omega_{1}+\omega_{2}\right)\right) \\
& \quad \times \gamma^{(2)}\left(-i\left(a_{i}+a_{j}\right)-i \omega_{2}\left(r-\left(u_{i}+u_{j}\right)\right) ;-i \omega_{2} r,-i\left(\omega_{1}+\omega_{2}\right)\right),
\end{aligned}
$$

with the balancing condition $i \sum_{i=1}^{6} a_{i}=\omega_{1}+\omega_{2}$ and $\sum_{i=1}^{6} u_{i}=0$, where $C=2 y^{2}-\sum_{i=1}^{6} u_{i}^{2}$.

As we add $\mu$ to first three coefficients and $\mathrm{z}$ variable, coming from the fundamental representation of the flavor group and gauge group, subtract $\mu$ from the last three coefficients, the left-hand side of the equation turns out to be

$$
\begin{aligned}
& \frac{1}{r \sqrt{-\omega_{1} \omega_{2}}} \sum_{y=0}^{[r / 2]} e^{\frac{\pi i c}{2}} \epsilon(y) \int_{-\mu}^{\infty} d z \prod_{i=1}^{3} \gamma^{(2)}\left(-i\left(a_{i}-z\right)-i \omega_{1}\left(u_{i}-y\right) ;-i \omega_{1} r,-i\left(\omega_{1}+\omega_{2}\right)\right) \\
& \left.\quad \times \gamma^{(2)}\left(-i\left(a_{i}-z\right)\right)-i \omega_{2}\left(r-\left(u_{i}-y\right)\right) ;-i \omega_{2} r,-i\left(\omega_{1}+\omega_{2}\right)\right) \\
& \quad \times \prod_{i=4}^{6} \gamma^{(2)}\left(-i\left(a_{i}+z\right)-i \omega_{1}\left(u_{i}+y\right) ;-i \omega_{1} r,-i\left(\omega_{1}+\omega_{2}\right)\right) \\
& \quad \times \gamma^{(2)}\left(-i\left(a_{i}+z\right)-i \omega_{2}\left(r-\left(u_{i}+y\right)\right) ;-i \omega_{2} r,-i\left(\omega_{1}+\omega_{2}\right)\right) \\
& \quad \times\left[\frac{\prod_{i=1}^{3} \gamma^{(2)}\left(-i\left(a_{i}+z+2 \mu\right)-i \omega_{1}\left(u_{i}+y\right) ;-i \omega_{1} r,-i\left(\omega_{1}+\omega_{2}\right)\right)}{\gamma^{(2)}\left(\mp 2 i(z+\mu) \mp i \omega_{1}(2 y) ;-i \omega_{1} r,-i\left(\omega_{1}+\omega_{2}\right)\right)}\right. \\
& \quad \times \frac{\gamma^{(2)}\left(-i\left(a_{i}+z+2 \mu\right)-i \omega_{2}\left(r-\left(u_{i}+y\right)\right) ;-i \omega_{2} r,-i\left(\omega_{1}+\omega_{2}\right)\right)}{\gamma^{(2)}\left(\mp 2 i(z+\mu)-i \omega_{2}(r \mp(2 y)) ;-i \omega_{2} r,-i\left(\omega_{1}+\omega_{2}\right)\right)} \\
& \quad \times \prod_{i=4}^{6} \gamma^{(2)}\left(-i\left(a_{i}-z-2 \mu\right)-i \omega_{1}\left(u_{i}-y\right) ;-i \omega_{1} r,-i\left(\omega_{1}+\omega_{2}\right)\right) \\
& \left.\quad \times \gamma^{(2)}\left(-i\left(a_{i}-z-2 \mu\right)-i \omega_{2}\left(r-\left(u_{i}-y\right)\right) ;-i \omega_{2} r,-i\left(\omega_{1}+\omega_{2}\right)\right)\right] .
\end{aligned}
$$

The main idea is to transform the gauge group from $S U(2)$ to $U(1)$. In order to achieve this goal we will use the asymptotic relations of the special function $\gamma^{(2)}\left(z ; \omega_{1}, \omega_{2}\right)$. Furthermore, as an outcome we will observe that there is a transformation in the flavor group as well. After the process we also have the following right-hand side:

$$
\begin{aligned}
= & \prod_{i=1}^{3} \prod_{j=4}^{6} \gamma^{(2)}\left(-i\left(a_{i}+a_{j}\right)-i \omega_{1}\left(u_{i}+u_{j}\right) ;-i \omega_{1} r,-i\left(\omega_{1}+\omega_{2}\right)\right) \\
& \times \gamma^{(2)}\left(-i\left(a_{i}+a_{j}\right)-i \omega_{2}\left(r-\left(u_{i}+u_{j}\right)\right) ;-i \omega_{2} r,-i\left(\omega_{1}+\omega_{2}\right)\right) \\
& \times\left[\prod_{1 \leq i<j \leq 3} \gamma^{(2)}\left(-i\left(a_{i}+a_{j}+2 \mu\right)-i \omega_{1}\left(u_{i}+u_{j}\right) ;-i \omega_{1} r,-i\left(\omega_{1}+\omega_{2}\right)\right)\right. \\
& \times \gamma^{(2)}\left(-i\left(a_{i}+a_{j}+2 \mu\right)-i \omega_{2}\left(r-\left(u_{i}+u_{j}\right)\right) ;-i \omega_{2} r,-i\left(\omega_{1}+\omega_{2}\right)\right) \\
& \times \gamma^{(2)}\left(-i\left(a_{i+3}+a_{j+3}-2 \mu\right)-i \omega_{1}\left(u_{i+3}+u_{j+3}\right) ;-i \omega_{1} r,-i\left(\omega_{1}+\omega_{2}\right)\right) \\
& \left.\times \gamma^{(2)}\left(-i\left(a_{i+3}+a_{j+3}-2 \mu\right)-i \omega_{2}\left(r-\left(u_{i+3}+u_{j+3}\right)\right) ;-i \omega_{2} r,-i\left(\omega_{1}+\omega_{2}\right)\right)\right] .
\end{aligned}
$$


Here, from the asymptotic relations which hyperbolic gamma functions satisfy, each term in the brackets behaves in a particular way:

$$
\begin{aligned}
& e^{\left(\frac{2 i \pi}{r}(\mu+z)\left(\omega_{1}^{-1}+\omega_{2}^{-1}\right)+2 i \pi y\right)} \gamma^{(2)}\left(\mp 2 i(z+\mu) \mp i \omega_{1}(2 y) ;-i \omega_{1} r,-i\left(\omega_{1}+\omega_{2}\right)\right)^{-1} \\
& \times \gamma^{(2)}\left(\mp 2 i(z+\mu) \mp i \omega_{2}(r-2 y) ;-i \omega_{2} r,-i\left(\omega_{1}+\omega_{2}\right)\right)^{-1} \stackrel{\mu \rightarrow \infty}{\longrightarrow} 1, \\
& \prod_{i=1}^{3} \gamma^{(2)}\left(-i\left(a_{i}+z+2 \mu\right)-i \omega_{1}\left(u_{i}+y\right) ;-i \omega_{1} r,-i\left(\omega_{1}+\omega_{2}\right)\right) \\
& \times \gamma^{(2)}\left(-i\left(a_{i}+z+2 \mu\right)-i \omega_{2}\left(r-\left(u_{i}+y\right)\right) ;-i \omega_{2} r,-i\left(\omega_{1}+\omega_{2}\right)\right) \\
& \times \prod_{i=4}^{6} \gamma^{(2)}\left(-i\left(a_{i}-z-2 \mu\right)-i \omega_{1}\left(u_{i}-y\right) ;-i \omega_{1} r,-i\left(\omega_{1}+\omega_{2}\right)\right) \\
& \times \gamma^{(2)}\left(-i\left(a_{i}-z-2 \mu\right)-i \omega_{2}\left(r-\left(u_{i}-y\right)\right) ;-i \omega_{2} r,-i\left(\omega_{1}+\omega_{2}\right)\right) \\
& \stackrel{\mu \rightarrow \infty}{\longrightarrow} \prod_{i=1}^{3} e^{\left[B_{2,2}\left(-i\left(a_{i}+z+2 \mu\right)-i \omega_{1}\left(u_{i}+y\right) ; \omega\right)+B_{2,2}\left(-i\left(a_{i}+z+2 \mu\right)-i \omega_{2}\left(r-\left(u_{i}+y\right)\right) ; \omega\right)\right]} \\
& \times \prod_{i=4}^{6} e^{\left[-B_{2,2}\left(-i\left(a_{i}-z-2 \mu\right)-i \omega_{1}\left(u_{i}-y\right) ; \omega\right)-B_{2,2}\left(-i\left(a_{i}-z-2 \mu\right)-i \omega_{2}\left(r-\left(u_{i}-y\right)\right) ; \omega\right)\right]}(1+o(1)), \\
& \prod_{1 \leq i<j \leq 3} \gamma^{(2)}\left(-i\left(a_{i}+a_{j}+2 \mu\right)-i \omega_{1}\left(u_{i}+u_{j}\right) ;-i \omega_{1} r,-i\left(\omega_{1}+\omega_{2}\right)\right) \\
& \times \gamma^{(2)}\left(-i\left(a_{i}+a_{j}+2 \mu\right)-i \omega_{2}\left(r-\left(u_{i}+u_{j}\right)\right) ;-i \omega_{2} r,-i\left(\omega_{1}+\omega_{2}\right)\right) \\
& \times \gamma^{(2)}\left(-i\left(a_{i+3}+a_{j+3}-2 \mu\right)-i \omega_{1}\left(u_{i+3}+u_{j+3}\right) ;-i \omega_{1} r,-i\left(\omega_{1}+\omega_{2}\right)\right) \\
& \times \gamma^{(2)}\left(-i\left(a_{i+3}+a_{j+3}-2 \mu\right)-i \omega_{2}\left(r-\left(u_{i+3}+u_{j+3}\right)\right) ;-i \omega_{2} r,-i\left(\omega_{1}+\omega_{2}\right)\right) \\
& \stackrel{\mu \rightarrow \infty}{\longrightarrow} \prod_{1 \leq i<j \leq 3} e^{\left[B_{2,2}\left(-i\left(a_{i}+a_{j}+2 \mu\right)-i \omega_{1}\left(u_{i}+u_{j}\right) ; \omega\right)+B_{2,2}\left(-i\left(a_{i}+a_{j}+2 \mu\right)-i \omega_{2}\left(r-\left(u_{i}+u_{j}\right)\right) ; \omega\right)\right]} \\
& \times \prod_{4 \leq i<j \leq 6} e^{\left[-B_{2,2}\left(-i\left(a_{i}+a_{j}-2 \mu\right)-i \omega_{1}\left(u_{i}+u_{j}\right) ; \omega\right)-B_{2,2}\left(-i\left(a_{i}+a_{j}-2 \mu\right)-i \omega_{2}\left(r-\left(u_{i}+u_{j}\right) ; \omega\right)\right]\right.}(1+o(1)) .
\end{aligned}
$$

Hence, after the reduction of integration we rename $a_{i+3}=b_{i}$ and $u_{i+3}=v_{i}$ obtain (2.7)

$$
\begin{aligned}
& \frac{1}{r \sqrt{-\omega_{1} \omega_{2}}} \sum_{y=0}^{[r / 2]} \epsilon(y) e^{\frac{\pi i c}{2}} \int_{-\infty}^{\infty} d z \prod_{i=1}^{3} \gamma^{(2)}\left(-i\left(a_{i}-z\right)-i \omega_{1}\left(u_{i}-y\right) ;-i \omega_{1} r,-i\left(\omega_{1}+\omega_{2}\right)\right) \\
& \quad \times \gamma^{(2)}\left(-i\left(a_{i}-z\right)-i \omega_{2}\left(r-\left(u_{i}-y\right)\right) ;-i \omega_{2} r,-i\left(\omega_{1}+\omega_{2}\right)\right) \\
& \quad \times \gamma^{(2)}\left(-i\left(b_{i}+z\right)-i \omega_{1}\left(v_{i}+y\right) ;-i \omega_{1} r,-i\left(\omega_{1}+\omega_{2}\right)\right) \\
& \quad \times \gamma^{(2)}\left(-i\left(b_{i}+z\right)-i \omega_{2}\left(r-\left(v_{i}+y\right)\right) ;-i \omega_{2} r,-i\left(\omega_{1}+\omega_{2}\right)\right) \\
& =\prod_{i, j=1}^{3} \gamma^{(2)}\left(-i\left(a_{i}+b_{j}\right)-i \omega_{1}\left(u_{i}+v_{j}\right) ;-i \omega_{1} r,-i\left(\omega_{1}+\omega_{2}\right)\right) \\
& \quad \times \gamma^{(2)}\left(-i\left(a_{i}+b_{j}\right)-i \omega_{2}\left(r-\left(u_{i}+v_{j}\right)\right) ;-i \omega_{2} r,-i\left(\omega_{1}+\omega_{2}\right)\right) .
\end{aligned}
$$

with the balancing conditions $\sum_{i} a_{i}+b_{i}=\omega_{1}+\omega_{2}$ and $\sum_{i} u_{i}+v_{i}=0$. The constant term is $C=-2 y+$ $\left(u_{1}+u_{2}+u_{3}-v_{1}-v_{2}-v_{3}\right)$. 


\section{APPENDIX C: STAR-TRIANGLE RELATION FOR $\boldsymbol{r}=2$}

We start by introducing two different gamma functions

$$
\begin{gathered}
\Gamma(z ; q, p)=\prod_{i, j=0}^{\infty} \frac{1-z^{-1} p^{i+1} q^{j+1}}{1-z p^{i} q^{j}} \\
\Gamma(z ; q, p, t)=\prod_{i, j, k=0}^{\infty}\left(1-z^{-1} p^{i+1} q^{j+1} t^{k+1}\right)\left(1-z p^{i} q^{j} t^{k}\right)
\end{gathered}
$$

for $|q|,|p|,|t|<1$ and $z \in \mathbb{C}^{*}$. Additionally, we have the following identity:

$$
\Gamma(q z ; p, q, t)=\Gamma(z ; p, t) \Gamma(z ; p, q, t) .
$$

We use the asymptotic relation between $\gamma^{(2)}\left(z ; \omega_{1}, \omega_{2}\right)$ and $\Gamma(z ; q, p)$ given as below

$$
\Gamma\left(e^{2 \pi i v z} ; e^{2 \pi i v \omega_{1}}, e^{2 \pi i v \omega_{2}}\right) \underset{v \rightarrow 0}{=} e^{-\pi i\left(2 z-\left(\omega_{1}+\omega_{2}\right)\right) / 24 v \omega_{1} \omega_{2}} \gamma^{(2)}\left(z ; \omega_{1}, \omega_{2}\right) .
$$

For a particular asymptotic relation

$$
\begin{aligned}
& \Gamma\left(z q^{y} ; q^{r}, q p\right) \Gamma\left(z p^{r-y}, p^{r}, q p\right) \\
& \underset{v \rightarrow 0}{=} \gamma^{(2)}\left(-i z-i \omega_{2}(r-y) ;-i \omega_{2} r,-i \omega_{1}-i \omega_{2}\right) \gamma^{(2)}\left(-i z-i \omega_{1} y ;-i \omega_{1} r,-i \omega_{1}-i \omega_{2}\right)
\end{aligned}
$$

where $q=e^{2 \pi i v \omega_{1}}$ and $p=e^{2 \pi i v \omega_{2}}$, we apply (C3)

$$
\begin{aligned}
\Gamma\left(z q^{y} ; q^{r}, q p\right) \Gamma\left(z p^{r-y}, p^{r}, q p\right) & =\frac{\Gamma\left(p^{r} z q^{y} ; q^{r}, p^{r}, q p\right)}{\Gamma\left(z q^{y} ; q^{r}, p^{r}, q p\right)} \frac{\Gamma\left(q^{r} z p^{r-y} ; q^{r}, p^{r}, q p\right)}{\Gamma\left(z p^{r-y} ; q^{r}, p^{r}, q p\right)} \\
& =\frac{\Gamma\left(z p^{r-y}(q p)^{y} ; q^{r}, p^{r}, q p\right)}{\Gamma\left(z p^{r-y} ; q^{r}, p^{r}, q p\right)} \frac{\Gamma\left(q^{y} z(q p)^{r-y} ; q^{r}, p^{r}, q p\right)}{\Gamma\left(z q^{y} ; q^{r}, p^{r}, q p\right)} \\
& =\Gamma\left(z p^{r-y}(q p)^{y-1} ; q^{r}, p^{r}\right) \Gamma\left(q^{y} z(q p)^{r-y-1} ; q^{r}, p^{r}\right) .
\end{aligned}
$$

If we consider to use the identity in (C3) only once, we observe the following asymptotic relation:

$$
\begin{aligned}
& \Gamma\left(z p^{r-y}(q p)^{y-1} ; q^{r}, p^{r}\right) \Gamma\left(q^{y} z(q p)^{r-y-1} ; q^{r}, p^{r}\right) \\
& \underset{v \rightarrow 0}{=} \gamma^{(2)}\left(-i z-i \omega_{1}(r-1)-i \omega_{2}(r-y-1) ;-i \omega_{1} r,-i \omega_{2} r\right) \\
& \quad \times \gamma^{(2)}\left(-i z-i \omega_{2}(r-1)-i \omega_{1}(y-1) ;-i \omega_{1} r,-i \omega_{2} r\right) .
\end{aligned}
$$

Moreover, for $y<r$ we use the identity several times and obtain the following form:

$$
\begin{aligned}
\Gamma\left(z q^{y} ; q^{r}, q p\right) & \Gamma\left(z p^{r-y}, p^{r}, q p\right)=\prod_{k=0}^{y-1} \Gamma\left(p^{r-y} z(q p)^{k} ; q^{r}, p^{r}\right) \prod_{s=0}^{r-y-1} \Gamma\left(q^{y} z(q p)^{s} ; q^{r}, p^{r}\right) \\
= & \prod_{v \rightarrow 0}^{y-1} \gamma_{k=0}^{(2)}\left(-i z-i \omega_{2}(r-y+k)-i k \omega_{1} ;-2 i \omega_{1},-i \omega_{2}\right) \\
& \times \prod_{s=0}^{r-y-1} \gamma^{(2)}\left(-i z-i \omega_{1}(y+s)-i s \omega_{2} ;-2 i \omega_{1},-2 i \omega_{2}\right) .
\end{aligned}
$$


For $r=2$, we calculate the cases $y=0$ and $y=1$ from $(\mathrm{C} 11)$ and derive the relation between $\gamma^{(2)}\left(z ; \omega_{1}, \omega_{2}\right)$ pairs as follow:

$$
\begin{aligned}
& \gamma^{(2)}\left(-i z-i \omega_{2}(2-y) ;-2 i \omega_{2},-i \omega_{1}-i \omega_{2}\right) \gamma^{(2)}\left(-i z-i \omega_{1} y ;-2 i \omega_{1},-i \omega_{1}-i \omega_{2}\right) \\
& \quad=\gamma^{(2)}\left(-i z-i \omega_{2}-i(1-y) \omega_{1} ;-2 i \omega_{1},-2 i \omega_{2}\right) \gamma^{(2)}\left(-i z-i y \omega_{1} ;-2 i \omega_{1},-2 i \omega_{2}\right) .
\end{aligned}
$$

Furthermore, we use (C12) to rewrite (1.1) explicitly,

$$
\begin{aligned}
\frac{1}{2 \sqrt{-\omega_{1} \omega_{2}}} \sum_{y=0}^{1} e^{\frac{\pi i}{2}\left(2 y-\sum_{i}\left(u_{i}-v_{i}\right)\right)} \int_{-\infty}^{\infty} d z \prod_{i=1}^{3} \gamma^{(2)}\left(-i\left(a_{i}-z\right)-i \omega_{1}\left(u_{i}-y\right) ;-2 i \omega_{1},-2 i \omega_{2}\right) \\
\quad \times \gamma^{(2)}\left(-i\left(a_{i}-z\right)-i \omega_{2}-i \omega_{2}\left(1-\left(u_{i}-y\right)\right) ;-2 i \omega_{2},-2 i \omega_{1}\right) \\
\quad \times \gamma^{(2)}\left(-i\left(b_{i}+z\right)-i \omega_{1}\left(v_{i}+y\right) ;-2 i \omega_{1},-2 i \omega_{2}\right) \\
\quad \times \gamma^{(2)}\left(-i\left(b_{i}+z\right)-i \omega_{2}-i \omega_{1}\left(1-\left(v_{i}+y\right)\right) ;-2 i \omega_{2},-2 i \omega_{1}\right) \\
=\prod_{i, j=1}^{3} \gamma^{(2)}\left(-i\left(a_{i}+b_{j}\right)-i \omega_{1}\left(u_{i}+v_{j}\right) ;-2 i \omega_{1},-2 i \omega_{2}\right) \\
\quad \times \gamma^{(2)}\left(-i\left(a_{i}+b_{j}\right)-i \omega_{2}-i \omega_{1}\left(1-\left(u_{i}+v_{j}\right)\right) ;-2 i \omega_{2},-2 i \omega_{1}\right) .
\end{aligned}
$$

Then we identify $-2 i \omega_{1}$ with $b,-2 i \omega_{2}$ with $1 / b$, redefine $u_{i}=\mu_{i}, v_{i}=1-\nu_{i}$ and all coefficients without $-2 i$ multiplier. Thus, the integral identity takes the form

$$
\begin{aligned}
& \sum_{\nu=0}^{1}(-1) \frac{2 \nu-3-\sum_{i}{ }_{i}^{\left(\mu_{i}-\nu_{i}\right)}}{2} \int_{-\infty}^{\infty} \frac{d x}{2 i} \prod_{i=1}^{3} \gamma^{(2)}\left(\frac{a_{i}+x+b\left(1-\nu_{i}-\nu\right)}{2} ; b, b^{-1}\right) \gamma^{(2)}\left(\frac{a_{i}+x+b^{-1}+b\left(\nu_{i}+\nu\right)}{2} ; b, b^{-1}\right) \\
& \quad \times \gamma^{(2)}\left(\frac{b_{i}-x+b\left(-\mu_{i}+\nu\right)}{2} ; b, b^{-1}\right) \gamma^{(2)}\left(\frac{\left(b_{i}-x+b^{-1}+b\left(1+\mu_{i}-\nu\right)\right)}{2} ; b, b^{-1}\right) \\
& =\prod_{i, j=1}^{3} \gamma^{(2)}\left(\frac{a_{i}+b_{j}+b\left(1-\nu_{i}-\mu_{j}\right)}{2} ; b, b^{-1}\right) \gamma^{(2)}\left(\frac{\left.a_{i}+b_{j}+b^{-1}+b\left(\nu_{i}+\mu_{j}\right)\right)}{2} ; b, b^{-1}\right) .
\end{aligned}
$$

This is exactly the integral identity (4.1).

[1] I. Gahramanov and S. Jafarzade, Integrable lattice spin models from supersymmetric dualities, Phys. Part. Nucl. Lett. 15, 650 (2018).

[2] M. Yamazaki, Integrability as duality: The Gauge/YBE correspondence, Phys. Rep. 859, 1 (2020).

[3] V. Spiridonov, Elliptic beta integrals and solvable models of statistical mechanics, Contemp. Math. 563, 181 (2012).

[4] A. P. Kels, New solutions of the star-triangle relation with discrete and continuous spin variables, J. Phys. A 48, 435201 (2015).

[5] I. Gahramanov and V. Spiridonov, The star-triangle relation and $3 \mathrm{~d}$ superconformal indices, J. High Energy Phys. 08 (2015) 040.

[6] A. P. Kels and M. Yamazaki, Elliptic hypergeometric sum/ integral transformations and supersymmetric lens index, SIGMA 14, 013 (2018).
[7] S. Jafarzade and Z. Nazari, A new integrable Ising-type model from $2 \mathrm{~d} \mathcal{N}=(2,2)$ dualities, arXiv:1709.00070.

[8] J. de-la Cruz-Moreno and H. Garca-Compen, Star-triangle type relations from $2 d \mathcal{N}=(0,2) \operatorname{USp}(2 N)$ dualities, J. High Energy Phys. 01 (2021) 023.

[9] I. Gahramanov and A. P. Kels, The star-triangle relation, lens partition function, and hypergeometric sum/integrals, J. High Energy Phys. 02 (2017) 040.

[10] R. Baxter, Exactly solved models in statistical mechanics (Academic Press, London, 1982).

[11] R. Baxter, Star-triangle and star-star relations in statistical mechanics, Int. J. Mod. Phys. B 11, 27 (1997).

[12] D. Gang, Chern-Simons theory on $L(p, q)$ lens spaces and localization, J. Korean Phys. Soc. 74, 1119 (2019).

[13] F. Benini, T. Nishioka, and M. Yamazaki, 4d index to 3d index and 2d TQFT, Phys. Rev. D 86, 065015 (2012). 
[14] Y. Imamura and D. Yokoyama, $S^{3} / Z_{n}$ partition function and dualities, J. High Energy Phys. 11 (2012) 122.

[15] Y. Imamura, H. Matsuno, and D. Yokoyama, Factorization of the $S^{3} / \mathbb{Z}_{n}$ partition function, Phys. Rev. D 89, 085003 (2014).

[16] F. Nieri and S. Pasquetti, Factorisation and holomorphic blocks in 4d, J. High Energy Phys. 11 (2015) 155.

[17] L. F. Alday, M. Fluder, and J. Sparks, The large N limit of M2-branes on lens spaces, J. High Energy Phys. 10 (2012) 057.

[18] M. Yamazaki, Four-dimensional superconformal index reloaded, Theor. Math. Phys. 174, 154 (2013).

[19] E. Eren, I. Gahramanov, S. Jafarzade, and G. Mogol, Gamma function solutions to the star-triangle equation, Nucl. Phys. B963, 115283 (2021).

[20] M. Honda, How to resum perturbative series in $3 \mathrm{~d} N=2$ Chern-Simons matter theories, Phys. Rev. D 94, 025039 (2016).

[21] A. Nedelin, F. Nieri, and M. Zabzine, $q$-Virasoro modular double and $3 \mathrm{~d}$ partition functions, Commun. Math. Phys. 353, 1059 (2017).

[22] V. V. Bazhanov, V. V. Mangazeev, and S. M. Sergeev, Faddeev-Volkov solution of the Yang-Baxter equation and discrete conformal symmetry, Nucl. Phys. B784, 234 (2007).

[23] V. V. Bazhanov, V. V. Mangazeev, and S. M. Sergeev, Exact solution of the Faddeev-Volkov model, Phys. Lett. A 372, 1547 (2008).

[24] S. Jafarzade, New pentagon identities revisited, J. Phys. Conf. Ser. 1194, 012054 (2019).

[25] L. Hadasz, M. Pawelkiewicz, and V. Schomerus, Self-dual continuous series of representations for $U_{-} q(\operatorname{sl}(2))$ and U_q(osp(1 - 2)), J. High Energy Phys. 10 (2014) 091.

[26] G. Sarkissian and V. P. Spiridonov, From rarefied elliptic beta integral to parafermionic star-triangle relation, J. High Energy Phys. 10 (2018) 097.

[27] L. F. Alday, D. Martelli, P. Richmond, and J. Sparks, Localization on three-manifolds, J. High Energy Phys. 10 (2013) 095.

[28] B. Willett, Localization on three-dimensional manifolds, J. Phys. A 50, 443006 (2017).

[29] F. Benini and W. Peelaers, Higgs branch localization in three dimensions, J. High Energy Phys. 05 (2014) 030.

[30] F. van de Bult et al., Hyperbolic hypergeometric functions, Ph.D. thesis, University of Amsterdam, Amsterdam Netherlands, 2007.

[31] A. Amariti, Integral identities for 3d dualities with $\mathrm{SP}(2 \mathrm{~N})$ gauge groups, arXiv:1509.02199.

[32] V. Spiridonov, Rarefied elliptic hypergeometric functions, Adv. Math. 331, 830 (2018).

[33] C. Cordova, B. Heidenreich, A. Popolitov, and S. Shakirov, Orbifolds and exact solutions of strongly-coupled matrix models, Commun. Math. Phys. 361, 1235 (2018).

[34] V. Spiridonov and G. Vartanov, Superconformal indices for $N=1$ theories with multiple duals, Nucl. Phys. B824, 192 (2010).

[35] I. Gahramanov and G. Vartanov, Extended global symmetries for 4D $N=1$ SQCD theories, J. Phys. A 46, 285403 (2013).
[36] O. Aharony, S. S. Razamat, N. Seiberg, and B. Willett, 3d dualities from 4d dualities, J. High Energy Phys. 07 (2013) 149.

[37] A. Karch, Seiberg duality in three-dimensions, Phys. Lett. B 405, 79 (1997).

[38] F. Dolan and H. Osborn, Applications of the superconformal index for protected operators and q-hypergeometric identities to $N=1$ dual theories, Nucl. Phys. B818, 137 (2009).

[39] N. Hama, K. Hosomichi, and S. Lee, SUSY gauge theories on squashed three-spheres, J. High Energy Phys. 05 (2011) 014.

[40] R. Kashaev, F. Luo, and G. Vartanov, A TQFT of TuraevViro type on shaped triangulations, Ann. Inst. Henri Poincaré 17, 1109 (2016).

[41] I. Gahramanov and H. Rosengren, A new pentagon identity for the tetrahedron index, J. High Energy Phys. 11 (2013) 128.

[42] I. Gahramanov and H. Rosengren, Integral pentagon relations for 3d superconformal indices, Proc. Symp. Pure Math. 93, 165 (2016).

[43] I. Gahramanov and H. Rosengren, Basic hypergeometry of supersymmetric dualities, Nucl. Phys. B913, 747 (2016).

[44] U. Pachner, P. L. homeomorphic manifolds are equivalent by elementary shellings, Eur. J. Combinatorics 12, 129 (1991).

[45] T. Dimofte, D. Gaiotto, and S. Gukov, Gauge theories labelled by three-manifolds, Commun. Math. Phys. 325, 367 (2014).

[46] S. Benvenuti and S. Pasquetti, $3 \mathrm{~d} \mathcal{N}=2$ mirror symmetry, PQ-webs and monopole superpotentials, J. High Energy Phys. 08 (2016) 136.

[47] D. N. Bozkurt and I. Gahramanov, Pentagon identities arising in supersymmetric gauge theory computations, Teor. Mat. Fiz. 198, 215 (2019).

[48] S. Alexandrov and B. Pioline, Theta series, wall-crossing and quantum dilogarithm identities, Lett. Math. Phys. 106, 1037 (2016).

[49] R. Kashaev, Eulers beta function and pentagon relations, Acta Mathematica Vietnamica 39, 561 (2014).

[50] F. Benini and S. Cremonesi, Partition functions of $\mathcal{N}=$ $(2,2)$ gauge theories on $\mathrm{S}^{2}$ and vortices, Commun. Math. Phys. 334, 1483 (2015).

[51] A. Zamolodchikov, 'Fishnet' diagrams as a completely integrable system, Phys. Lett. 97B, 63 (1980).

[52] V. V. Bazhanov, A. P. Kels, and S. M. Sergeev, Quasiclassical expansion of the star-triangle relation and integrable systems on quad-graphs, J. Phys. A 49, 464001 (2016).

[53] P. Kulish, Quantum superalgebra Osp(2-1), J. Sov. Math. 54, 923 (1991).

[54] P. Kulish and N. Reshetikhin, Universal R matrix of the quantum superalgebra osp(2-1), Lett. Math. Phys. 18, 143 (1989).

[55] H. Saleur, Quantum Osp $(1,2)$ and solutions of the graded Yang-Baxter equation, Nucl. Phys. B336, 363 (1990).

[56] M. Pawelkiewicz, V. Schomerus, and P. Suchanek, The universal Racah-Wigner symbol for $\mathrm{U}_{q}(\operatorname{osp}(1-2))$, J. High Energy Phys. 04 (2014) 079. 
[57] H. Poghosyan and G. Sarkissian, Comments on fusion matrix in $N=1$ super Liouville field theory, Nucl. Phys. B909, 458 (2016).

[58] J. Teschner and G. Vartanov, 6j symbols for the modular double, quantum hyperbolic geometry, and supersymmetric gauge theories, Lett. Math. Phys. 104, 527 (2014).

[59] L. Hadasz, On the fusion matrix of the $N=1$ NeveuSchwarz blocks, J. High Energy Phys. 12 (2007) 071.

[60] R. M. Kashaev, Heisenberg double and the pentagon relation, St. Petersburg Math. J. 8, 585 (1997).

[61] L. D. Faddeev, Modular double of quantum group, Math. Phys. Stud. 21, 149 (2000).

[62] K. Hikami, Hyperbolic structure arising from a knot invariant, Int. J. Mod. Phys. A 16, 3309 (2001).

[63] J. E. Andersen and R. Kashaev, Complex quantum ChernSimons, arXiv:1409.1208.
[64] K. Hikami, Generalized volume conjecture and the a-polynomials: The Neumann-Zagier potential function as a classical limit of the partition function, J. Geom. Phys. 57, 1895 (2007).

[65] K. Hikami and R. Inoue, Braiding operator via quantum cluster algebra, J. Phys. A 47, 474006 (2014).

[66] C.-T. Chan, A. Mironov, A. Morozov, and A. Sleptsov, Orthogonal polynomials in mathematical physics, Rev. Math. Phys. 30, 1840005 (2018).

[67] R. Kashaev, The Yang-Baxter relation and gauge invariance, J. Phys. A 49, 164001 (2016).

[68] L. Faddeev, Discrete Heisenberg-Weyl group and modular group, Lett. Math. Phys. 34, 249 (1995).

[69] S. Woronowicz, Quantum exponential function, Rev. Math. Phys. 12, 873 (2000). 